\title{
Developmental Hypoxia Has Negligible Effects on Long-Term Hypoxia Tolerance and Aerobic Metabolism of Atlantic Salmon (Salmo salar)
}

\author{
Andrew T. Wood ${ }^{1,2, *}$ \\ Timothy D. Clark ${ }^{1,2}$ \\ Sarah J. Andrewartha ${ }^{1,2}$ \\ Nicholas G. Elliott ${ }^{1}$ \\ Peter B. Frappell ${ }^{2}$ \\ ${ }^{1}$ Commonwealth Scientific and Industrial Research \\ Organisation Agriculture and Food, 3-4 Castray Esplanade, \\ Battery Point, Tasmania, Australia; ${ }^{2}$ Institute for Marine and \\ Antarctic Studies, University of Tasmania, 20 Castray \\ Esplanade, Battery Point, Tasmania, Australia
}

Accepted 3/6/2017; Electronically Published 5/1/2017

\begin{abstract}
Exposure to developmental hypoxia can have long-term impacts on the physiological performance of fish because of irreversible plasticity. Wild and captive-reared Atlantic salmon (Salmo salar) can be exposed to hypoxic conditions during development and continue to experience fluctuating oxygen levels as juveniles and adults. Here, we examine whether developmental hypoxia impacts subsequent hypoxia tolerance and aerobic performance of Atlantic salmon. Individuals at $8^{\circ} \mathrm{C}$ were exposed to $50 \%$ (hypoxia) or $100 \%$ (normoxia) dissolved oxygen (DO) saturation (as percent of air saturation) from fertilization for $\sim 100 \mathrm{~d}$ (800 degree days) and then raised in normoxic conditions for a further $15 \mathrm{mo}$. At $18 \mathrm{mo}$ after fertilization, aerobic scope was calculated in normoxia $(100 \%$ DO) and acute $(18 \mathrm{~h}$ ) hypoxia (50\% DO) from the difference between the minimum and maximum oxygen consumption rates $\left(\dot{\mathrm{M}}_{2 \text { min }}\right.$ and $\dot{\mathrm{MO}}_{2 \text { max }}$, respectively) at $10^{\circ} \mathrm{C}$. Hypoxia tolerance was determined as the $\mathrm{DO}$ at which loss of equilibrium (LOE) occurred in a constantly decreasing DO environment. There was no difference in $\dot{\mathrm{M}}_{2 \text { min }}, \dot{\mathrm{M}}_{2 \text { max }}$, or aerobic scope between fish raised in hypoxia or normoxia. There was some evidence that hypoxia tolerance was lower (higher DO at LOE) in hypoxiaraised fish compared with those raised in normoxia, but the magnitude of the effect was small (12.52\% DO vs. $11.73 \%$ DO at LOE). Acute hypoxia significantly reduced aerobic scope by reducing $\dot{\mathrm{M}}_{2 \text { max }}$, while $\dot{\mathrm{M}}_{2 \text { min }}$ remained unchanged. Interestingly, acute hypoxia uncovered individual-level relationships between DO at LOE and $\dot{M}_{2 \text { min }}, \dot{M}_{2 \text { max }}$, and aerobic scope. We discuss our findings in the context of developmental tra-
\end{abstract}

*Corresponding author; e-mail: andrew.wood@csiro.au.

Physiological and Biochemical Zoology 90(4):494-501. 2017. (C) 2017 by The University of Chicago. All rights reserved. 1522-2152/2017/9004-6148\$15.00 DOI: $10.1086 / 692250$ jectories and the role of aerobic performance in hypoxia tolerance.

Keywords: hypoxia, aerobic metabolism, hypoxia tolerance, developmental trajectory.

\section{Introduction}

Developmental plasticity in response to environmental stressors can have long-term consequences for individual performance because of impacts on developmental trajectories (Monaghan 2008; Burggren and Reyna 2011; Mueller et al. 2015; Garland et al. 2017). For example, a hypoxic developmental rearing environment can impact cardiovascular development and regulation in fish (Pelster 2002; Bagatto 2005; Miller et al. 2011). Such responses to environmental conditions during development are of increasing concern in the current era of environmental change, where hypoxic events are predicted to become more widespread (Diaz and Rosenberg 2008; Altieri and Gedan 2015).

Salmonid embryos and yolk sac alevins often experience hypoxia during development in both wild redds and aquaculture hatcheries. The availability of oxygen to the embryos is affected by many factors, including ambient dissolved oxygen (DO) levels, water flow characteristics, embryo density, redd depth, sediment type, and developmental stage (Ingendahl 2001; Youngson et al. 2004; Ciuhandu et al. 2007; Greig et al. 2007; Miller et al. 2008; Dhiyebi et al. 2013). While hatchery water conditions are typically monitored and controlled, oxygen levels within incubators can still become hypoxic in crowded conditions (McLean and Lim 1985). Localized hypoxic zones can develop within commercial systems (e.g., Heath stack tray incubators) during later developmental stages (A. T. Wood, unpublished data). Wild and cultured salmonids are also likely to experience fluctuating DO levels during postlarval rearing. For example, salmonids reared in marine sea cage aquaculture can experience significant DO variations over temporal and spatial scales (Johansson et al. 2006; Oppedal et al. 2011; Burt et al. 2012).

Hypoxia can limit aerobic capacity because of reduced oxygen supply, which can decrease activity levels, growth, and survival if threshold oxygen levels are breached (Pedersen 1987; Wang et al. 2009). Fish that are able to maintain a higher maximum oxygen consumption rate $\left(\dot{\mathrm{M}}_{2 \max }\right)$ over a range of oxygen levels may have a higher aerobic performance in low oxygen conditions. Hypoxia tolerance may also impact survival when DO levels fall below the critical oxygen tension $\left(\mathrm{O}_{2 \text { crit }}\right)$ required to maintain the standard metabolic rate (minimum oxygen con- 
sumption rate $\left.\left[\dot{\mathrm{M}}_{2 \text { min }}\right]\right)$. Survival below $\mathrm{O}_{2 \text { crit }}$ is dependent on anaerobic performance and metabolic depression, and most fish cannot survive for prolonged periods under such conditions (Claireaux and Chabot 2016).

Fish show a range of responses to developmental hypoxia. For example, rainbow trout (Oncorhynchus mykiss) have lower maximum sustained swimming speeds at $65-110 \mathrm{~d}$ postfertilization after being reared from 0 to $57 \mathrm{~d}$ postfertilization in hypoxic conditions of 34\% DO (percent of air saturation; Johnston et al. 2013). Moreover, $\mathrm{Mo}_{2}$ min below the $\mathrm{O}_{2 \text { crit }}$ of $4 \mathrm{~d}$ postfertilization zebrafish (Danio rerio) was higher for individuals exposed to $5 \%$ $\mathrm{DO}$ for $4 \mathrm{~h}$ at 24 and $36 \mathrm{~h}$ after fertilization, but $\dot{\mathrm{Mo}}_{2 \text { min }}$ returned to control levels after approximately $6 \mathrm{mo}$ in normoxia, indicating that the response was plastic and reversible (Robertson et al. 2014). On the other hand, exposure to $40 \%$ DO from 30 to $38 \mathrm{~d}$ after hatch did not change the DO at loss of equilibrium (LOE) of 1-yr-old European sea bass (Dicentrarchus labrax) compared with animals reared in normoxia (Vanderplancke et al. 2014). Clearly, a greater research effort is required to decipher any consistent, long-term physiological responses to developmental hypoxia in fish.

Here, we investigated the long-term impacts of embryonic and larval developmental oxygen levels ( $50 \%$ DO vs. $100 \%$ DO) on the aerobic performance and acute hypoxia tolerance of captive-reared Atlantic salmon (Salmo salar). At 18 mo after fertilization, $\dot{M}_{2 \text { min }}, \dot{M}_{2 \text { max }}$, and aerobic scope were measured in normoxia and acute hypoxia (50\% DO) to determine any lasting impacts on aerobic performance. The DO level at LOE was measured as an indicator of hypoxia tolerance. We also tested for interindividual relationships between hypoxia tolerance and $\dot{\mathrm{M}}_{2 \text { min }}, \dot{\mathrm{MO}}_{2 \text { max }}$, and aerobic scope. We hypothesized that developmental hypoxia may result in an increase in hypoxia tolerance and aerobic performance $\left(\dot{\mathrm{M}}_{2 \text { max }}\right) 18$ mo after fertilization because of irreversible phenotypic plasticity causing an increase in oxygen transport capacity.

\section{Methods}

Embryonic and Larval Incubation

Initial stages of the experiments were conducted at the SALTAS aquaculture facility (Wayatinah, Tasmania). Four half-sibling Atlantic salmon families were created by fertilizing eggs from four females with milt from one male, using captive-bred broodstock reared in freshwater at SALTAS. Half-sibling families were used to reduce potential variability in metabolic measurements and hypoxia tolerance between families (Anttila et al. 2013). Fertilized eggs were randomly allocated to two replicate mesh isolation baskets $(18 \mathrm{~cm} \times 14.5 \mathrm{~cm} \times 5.5 \mathrm{~cm})$ per treatment, with each replicate incubated in separate Heath trays $(39 \mathrm{~cm} \times 32 \mathrm{~cm} \times 5.5 \mathrm{~cm}$; Marisource). Each Heath tray held four isolation baskets from this study and an unrelated parallel study, with approximately 1,750 eggs per basket (7,000 eggs per Heath tray).

Eggs were incubated from fertilization through hatching ( $~ 520$ degree days [DD], $\sim 65 \mathrm{~d}$ ) to near yolk sac absorption ( 800 $\mathrm{DD}, \sim 100 \mathrm{~d})$ at $7.98^{\circ} \pm 0.15^{\circ} \mathrm{C}( \pm \mathrm{SD})$ at either $100.0 \% \pm 1.8 \%$
DO (as percent of air saturation; control) or $50.1 \% \pm 0.8 \%$ DO (hypoxia) in drum-screened raw river water in a flow-through system. Hypoxia exposure of 50\% DO was chosen as a suitably stressful treatment on the basis of previous studies of salmonids (Rombough 2007; Polymeropoulos 2013). The Heath tray system was modified to ensure consistent exposure to the experimental conditions by supplying each tray with an independent water supply at a rate of $10 \mathrm{~L} \mathrm{~min}^{-1}$. The temperature and DO of the two treatments were controlled by an OxyGuard Pacific monitoring system (OxyGuard, Denmark) with a submersible heater and nitrogen or oxygen injection into $200-\mathrm{L}$ treatment sumps. The nitrogen injection system failed on three occasions throughout the $100-\mathrm{d}$ incubation period, such that DO rose to normoxic levels for a maximum continuous period of $41 \mathrm{~h}$ before being rectified. Nonetheless, eggs in the hypoxia treatment spent $97 \%$ of their incubation between $45 \%$ and $55 \%$ DO (grand mean $51.1 \% \pm 7.6 \%$ DO when including nitrogen system failures). Eggs and alevins were reared according to best industry practice, which includes formalin treatments of 1.5$2 \mathrm{~mL} \mathrm{~L}^{-1}$ for $15 \mathrm{~min}$ three times weekly from 70 to $340 \mathrm{DD}$ (to prevent fungal growth) and removal of dead eggs and alevins from 280 to $950 \mathrm{DD}$. Additionally, at the eyed stage (280 DD), eggs were physically agitated to assist in the removal of dead embryos.

\section{Postlarval Rearing}

From $\sim 100 \mathrm{~d}$ after fertilization (800 DD), all treatment groups were returned to normoxic conditions, and at 4 mo after fertilization (980 DD), all fish were transferred from Heath trays into 50-L tanks for their first feeding. The two replicate isolation baskets were combined into one tank for each treatment. The two 50-L tanks were contained within a semiclosed recirculating system at SALTAS, supplied with drum-screened river water at ambient temperature. DO was maintained above $90 \%$ by supplementary oxygenation controlled by an OxyGuard Atlantic oxygen monitoring system (OxyGuard, Denmark). Fish were held in 50-L tanks from 4 mo (980 DD) to 8 mo after fertilization, after which they were transferred into two 500-L tanks on the same recirculating system until 17 mo after fertilization. Ambient temperature followed seasonal cycles and ranged from approximately $5^{\circ}$ to $15^{\circ} \mathrm{C}$. While replicate tanks were logistically impossible during this on-farm phase of the experiment, all fish were exposed to the same recirculating water, and there was no evidence that this approach represented a confounding factor in the results (e.g., mortality was negligible, and growth rates were the same between tanks).

Fish were transferred to Commonwealth Scientific and Industrial Research Organisation Laboratories (Hobart, Tasmania) at 17 mo after fertilization, $37 \mathrm{~d}$ before experiments. Fish were internally tagged with passive integrated transponder (PIT) tags $20 \mathrm{~d}$ before experiments and allowed to recover for $7 \mathrm{~d}$ before the treatment groups were mixed equally between two 200-L tanks within a recirculating freshwater filtration system. Temperature was maintained at $\sim 10^{\circ} \mathrm{C}$ and $\mathrm{DO}$ at $90 \%-100 \%$ by aeration. Fish were fed to satiation daily with commercial pellet food for $13 \mathrm{~d}$ 
before commencing respirometry and until completion of all experiments (except before experimental protocols).

\section{Respirometry}

The oxygen consumption rates $\left(\mathrm{Mo}_{2}\right.$; aerobic metabolic rates) of individual fish ( 105 g) were measured in 4.05-L (total volume) intermittent-flow respirometers containing freshwater and using practices outlined by Clark et al. (2013). Briefly, each respirometer consisted of a plastic chamber with an O-ring sealed lid through which water was continuously circulated by an external recirculation loop (13-mm-diameter tubing) and inline pump to ensure mixing. Fresh water was introduced into each respirometer during each flush cycle by a large submersible pump connected to each respirometer with polyvinyl chloride tubing and flushed out through a polyethylene standpipe vented $10 \mathrm{~cm}$ above the water surface to prevent backflow. A timercontrolled solenoid valve regulated water flow from the flush pump to produce a 12.5:7.5 min flush: seal cycle, which continuously repeated throughout all $\dot{\mathrm{M}}_{2}$ measurements unless stated otherwise. Ten respirometry chambers were submerged in a single water bath that was maintained at $\sim 10^{\circ} \mathrm{C}$ with a digitally controlled electric submersible titanium heater (Aqua Logic, San Diego, CA). DO was maintained at either $100 \%$ by aeration or $50 \%$ by injecting nitrogen (controlled by an OxyGuard Atlantic oxygen monitoring system). Oxygen concentration within each respirometry chamber was measured at 5-s intervals using an optical oxygen sensor sealed in the recirculation loop and connected to a four-channel FireSting $\mathrm{O}_{2}$ optical oxygen meter (Pyro Science, Aachen).

The rate of declining DO $\left(\% \mathrm{~min}^{-1}\right)$ during each 7.5 -min sealed cycle was determined by least squares regression, and $\dot{\mathrm{MO}}_{2}$ was calculated using the formula

$\dot{\mathrm{M}}_{2}=\frac{\Delta \mathrm{DO}}{\Delta t} \times\left[P_{\mathrm{B}}-\left(P_{\mathrm{S}} \times h\right)\right] \times \beta_{\mathrm{O}_{2}} \times$ volume $\times 0.2094$, where DO is the fractional DO saturation, $t$ is time in minutes, $P_{\mathrm{B}}$ is barometric pressure $(\mathrm{kPa}), P_{\mathrm{S}}$ is the calculated saturation vapor pressure of water $(\mathrm{kPa}$; Antoine equation), $h$ is the fractional relative humidity, $\beta_{\mathrm{O}_{2}}$ is the oxygen capacitance of water ( $0.5375 \mathrm{mg} \mathrm{L}^{-1} \mathrm{kPa}^{-1}$; see Dejours 1981), and volume is the volume of the respirometry chamber minus fish volume (L; assuming $1 \mathrm{~kg}$ wet fish mass $=1 \mathrm{~L}$ volume).

Metabolic measurements were conducted over eight consecutive days (10 fish per day), alternating daily between measurements in normoxia and hypoxia. Each fish was used for respirometry only once. Fish were fasted for at least $18 \mathrm{~h}$ before respirometry and were identified via PIT tag and weighed (Ohaus Scout Pro digital balance, Ohaus) before being transferred to respirometry chambers. Once sealed in the chambers, either the fish remained in normoxic water or they recovered from handling for $3 \mathrm{~h}$ before the DO was reduced to $50 \%$ over 1-2 h. Oxygen consumption rates were measured using a 12.5:7.5 min flush: seal cycle for 14.5-16 h with no disruption to ensure minimum $\mathrm{Mo}_{2}\left(\mathrm{Mo}_{2}\right.$ min $)$ was reached. Minimum $\mathrm{M}_{2}$ was determined for each individual as the mean of the lowest six $\mathrm{MO}_{2}$ measurements during the 14.5-16-h resting period (40-70 $\mathrm{Mo}_{2}$ measurements, depending on individual), excluding any $\dot{\mathrm{MO}}_{2}$ value outside $\pm 2 \mathrm{SD}$ of the mean (Clark et al. 2013; Norin et al. 2014). Given that fish were measured for at least $14.5 \mathrm{~h}$ in a postabsorptive state, it was assumed that $\dot{\mathrm{M}}_{2}$ min provided a reasonable estimation of standard metabolic rate (Chabot et al. 2016).

Maximum metabolic rate $\left(\dot{\mathrm{M}}_{2 \text { max }}\right)$ was subsequently measured in each fish, using an exhaustive exercise protocol. Fish were individually transferred from their respirometer to a 33-L cylindrical tank receiving water from the respirometry water bath at either $100 \%$ or $50 \%$ DO. Each fish was chased for 2 min by hand, tapping the tail as necessary to encourage continuous swimming, and all fish ceased continuous-burst swimming during the 2-min protocol. At the end of exercise, each fish was immediately (within $15 \mathrm{~s}$ ) placed back into the same sealed respirometer from which it came. The oxygen decline in the respirometers was measured until DO had decreased by a maximum of $15 \%$, and $\dot{M}_{2 \text { max }}$ was calculated from the steepest slope in any $~ 5$-min period during this time (e.g., see Norin and Clark 2016). On completion of all $\mathrm{Mo}_{2 \text { max }}$ measurements, all fish were returned to their respective holding tanks for at least $6 \mathrm{~d}$ until required for hypoxia tolerance experiments.

\section{Acute Hypoxia Tolerance}

The hypoxia tolerance of groups of 17-22 salmon (approximately half from each developmental treatment group) was tested in four hypoxia challenges conducted across $4 \mathrm{~d}$. After being fasted for $18 \mathrm{~h}$, fish were placed in a 200-L flow-through $\left(0.1 \mathrm{~L} \mathrm{~min}^{-1}\right)$ tank of freshwater at $10^{\circ} \mathrm{C}$ and recovered from handling overnight at $>90 \%$ DO. DO was measured at 5 -s intervals using an optical oxygen sensor (FireSting $\mathrm{O}_{2}$ oxygen meter, Pyro Science). In the morning, the DO was decreased at a rate of $4 \%-4.5 \% \mathrm{~min}^{-1}$ until $45 \% \mathrm{DO}$ was reached and then at $0.3 \%-0.35 \% \mathrm{~min}^{-1}$ thereafter by bubbling nitrogen at a controlled rate. The two different rates of DO decline were used to decrease the length of the experiment while still allowing a precise measure of the DO at LOE for each individual. LOE was defined as the DO where a fish could no longer maintain balance and their ventral surface was visible for $5 \mathrm{~s}$. At LOE, individuals were identified by PIT tag and transferred to a recovery tank. At the completion of each run, the fish were killed by anaesthetic overdose (Aqui-S, Lower Hutt, New Zealand) and their length and mass measured.

\section{Statistical Analyses}

Statistical analyses were performed using R (R Development Core Team 2015). Differences in $\dot{\mathrm{M}}_{2 \text { min }}, \dot{\mathrm{M}}_{2 \text { max }}$, and aerobic scope between developmental treatment groups (normoxia, hypoxia) and acute measurement DO (50\%, 100\%) were tested using two-way ANCOVA (type III sum of squares [SS]) including fish mass as the covariate. For $\dot{M}_{2 \text { max }}$ and aerobic scope, there was a significant interaction involving the covariate, which prevented robust comparisons of main effects. As such, log-log 
transformations of continuous variables were performed to meet the assumptions of an ANCOVA requiring homogeneity of slopes for between-group comparisons. Comparisons of mean DO at LOE between developmental treatment groups (normoxia, hypoxia) and acute measurement DO (50\%, 100\%) were carried out using a two-way ANOVA (type III SS). Linear regressions of mass-specific $\dot{\mathrm{M}}_{2 \text { min }}, \dot{\mathrm{M}}_{2 \text { max }}$, and aerobic scope against DO at LOE were calculated separately for each acute measurement DO group (50\%, 100\%).

\section{Results}

Mass

Mean fish mass did not differ between developmental treatment groups and averaged $104.5 \pm 2.7$ and $107.5 \pm 2.8 \mathrm{~g}$ at the $\dot{\mathrm{MO}}_{2}$ and LOE measurements, respectively $\left(\dot{\mathrm{MO}}_{2}: F_{1,78}=3.07\right.$, $P=0.084$; LOE: $F_{1,75}=2.18, P=0.14$ ).

\section{Metabolic Rates}

Positive linear relationships existed between fish mass and $\dot{\mathrm{M}}_{2 \text { min }}, \dot{\mathrm{M}}_{2 \text { max }}$, and aerobic scope (fig. $1 A-1 C$ ). Developmental oxygen level did not influence $\mathrm{Mo}_{2 \text { min }}, \dot{M}_{2 \text { max }}$, or aerobic scope when measured in either $50 \%$ DO or $100 \%$ DO (fig. $1 ; \dot{M}_{2 \min }: F_{1,75}=0.437, P=0.51 ; \dot{M}_{2 \max }: F_{1,75}=3.48$, $P=0.066$; aerobic scope: $F_{1,75}=2.48, P=0.12$ ). Thus, $\dot{M}_{2}$ values of developmental groups are combined herein but are presented separately in figures for clarity.

The $\dot{M}_{2}$ max and aerobic scope of fish measured at 50\% DO were $\sim 50 \%$ and $70 \%$ lower, respectively, than fish that were measured in $100 \% \mathrm{DO}$ (fig. $1 \mathrm{~B}, 1 \mathrm{E}$; $\dot{\mathrm{M}}_{2 \max }: F_{1,75}=1,343.1, P<$ 0.001; fig. $1 C, 1 F$; aerobic scope: $\left.F_{1,75}=633.7, P<0.001\right)$. However, $\dot{M}_{2}$ min was unaffected (fig. $1 A, 1 D ; \dot{M}_{2 \text { min }}: F_{1,75}=$ $0.158, P=0.69)$. There was a significant interaction between fish mass and measurement DO (50\% or $100 \%$ DO) for $\dot{M}_{2 \text { max }}$ and aerobic scope; the slopes of the relationships between mass and $\dot{\mathrm{M}}_{2 \text { max }}$ and between mass and aerobic scope (but not $\dot{\mathrm{MO}}_{2 \text { min }}$ ) were reduced in $50 \%$ DO compared with $100 \%$ DO (fig. $1 B, 1 C$; $\dot{M}_{2 \text { max }}: F_{1,72}=49.02, P<0.001$; aerobic scope: $F_{1,72}=34.8$, $P<0.001)$. Nevertheless, log-log transforming the relationships for $\dot{M}_{2 \max }$ and aerobic scope against mass revealed that the scaling exponent (slope) was not different between $50 \%$ and $100 \%$ DO levels. That is, the relative but not the absolute decrease in $\dot{\mathrm{MO}}_{2 \text { max }}$ and aerobic scope with acute hypoxia was proportional across body mass (inset in fig. $1 B, 1 C ; \dot{M}_{2 \max }: b=0.893, P<$ 0.001 ; aerobic scope: $b=0.759, P<0.001$ ).

\section{Acute Hypoxia Tolerance}

When combining $\mathrm{MO}_{2}$ measurements for both DO measurement groups, DO at LOE was slightly but significantly higher in the hypoxia-incubated developmental group $(12.52 \% \pm 0.27 \%$ DO) compared with the normoxia-incubated group (fig. 2; $\left.11.73 \% \pm 0.27 \% \mathrm{DO} ; F_{1,75}=4.11, P=0.046\right)$. There was no relationship between fish mass and DO at $\operatorname{LOE}\left(F_{1,72}=0.063\right.$, $P=0.80$ ). Additionally, the $\mathrm{Mo}_{2}$ measurement $\mathrm{DO}$ (50\% or
$100 \%$ DO) had no subsequent effect on DO at LOE (50\%: $11.81 \% \pm 0.27 \% \mathrm{DO} ; 100 \%: 12.47 \% \pm 0.27 \% \mathrm{DO} ; F_{1,75}=2.83$, $P=0.096)$.

\section{Relationship between $\dot{M O}_{2}$ and Hypoxia Tolerance}

When $\mathrm{Mo}_{2}$ was measured in normoxia, there was no relationship between DO at LOE and $\dot{\mathrm{M}}_{2 \text { min }}, \dot{\mathrm{M}}_{2 \text { max }}$, or aerobic scope (fig. $3 ; \dot{M}_{2 \text { min }}: F_{1,37}=0.12, R^{2}=0.003, P=0.73 ; \dot{M}_{2 \text { max }}: F_{1,37}=$ 0.016, $R^{2}=0.00, P=0.90$; aerobic scope: $F_{1,37}=0.065, R^{2}=$ $0.001, P=0.80$ ). Interestingly, measuring $\mathrm{MO}_{2}$ in hypoxia revealed significant relationships between $\mathrm{DO}$ at $\mathrm{LOE}$ and $\mathrm{Mo}_{2}$ min, $\dot{M}_{2 \text { max }}$, and aerobic scope (fig. $3 ; \dot{M}_{2 \text { min }}: F_{1,36}=7.77, R^{2}=0.18$, $P<0.01 ; \dot{M}_{2 \max }: F_{1,36}=12.73, R^{2}=0.26, P=0.001$ : aerobic scope: $\left.F_{1,36}=24.18, R^{2}=0.40, P<0.001\right)$.

\section{Discussion}

This study is one of only a few measuring the long-term impacts of developmental hypoxia on fish following an extended period of normoxia after incubation. We found no evidence that developmental hypoxia exposure during embryonic and larval incubation affects long-term aerobic performance in Atlantic salmon and only weak evidence for an effect on acute hypoxia tolerance.

The $\dot{M}_{2 \text { min }}, \dot{M}_{2 \text { max }}$, or aerobic scope did not differ between groups of Atlantic salmon raised in hypoxia or normoxia during development when measured in normoxia or hypoxia $\sim 18$ mo after fertilization. Given the link between aerobic metabolic attributes and performance traits, it is possible that our findings translate to parameters beyond what we have measured here. For example, it is possible that performance traits that are related to $\dot{\mathrm{M}}_{2 \text { max }}$ and aerobic scope (e.g., maximum swimming speed) and those related to $\dot{M}_{2 \text { min }}$ (e.g., growth and behavioral traits) may also suffer no long-term impacts of developmental hypoxia, but these ideas remain to be thoroughly investigated (Reidy et al. 2000; Metcalfe et al. 2016; Norin et al. 2016).

Somewhat counterintuitively, we found some evidence that salmon exposed to developmental hypoxia had decreased acute hypoxia tolerance (higher DO at LOE) compared with those raised in normoxia (fig. 2). Nonetheless, while the acute hypoxia tolerance of the two developmental incubation groups was significantly different $(P=0.046)$, the magnitude of the effect was small (12.52\% DO vs. $11.73 \%$ DO). For our experiment where DO was decreased at $0.33 \% \mathrm{~min}^{-1}$, this represents a difference in mean time to LOE of $\sim 2.4 \mathrm{~min}$. Such a difference may be negligible because the median time to LOE can vary substantially (up to $114.8 \mathrm{~min}$ ) between families of Atlantic salmon (Anttila et al. 2013). Thus, it is unclear what real-world impacts an effect of such small magnitude would have on fish performance and survival, given the spatial and temporal DO variability often experienced in wild and aquaculture environments.

The concept of developmental trajectories proposes that exposure to an environmental stressor such as hypoxia can cause plasticity during development, which may permanently impact the phenotype of an animal or be reversible following a return 

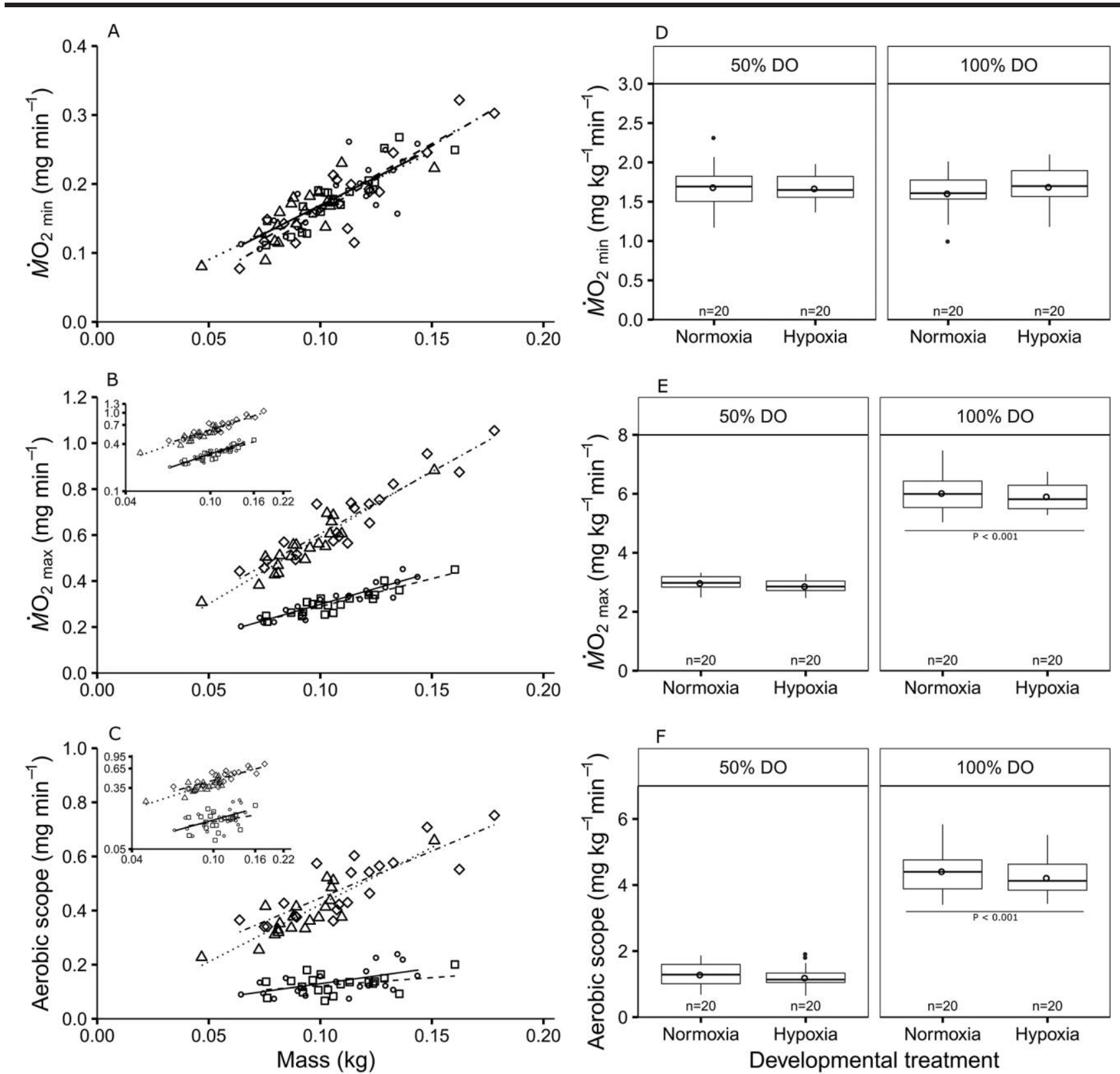

\section{Developmental treatment, $\dot{M} \mathrm{O}_{2} \mathrm{DO}$

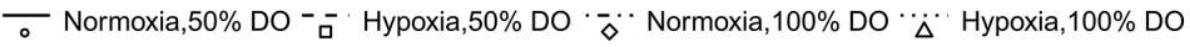

Figure 1. A-C, Relationship between fish mass and $\dot{\mathrm{M}}_{2 \min }(A), \dot{\mathrm{Mo}}_{2 \max }(B)$, and aerobic scope $(C)$ for each developmental oxygen treatment and $\mathrm{MO}_{2}$ dissolved oxygen (DO) measurement level. Points represent individuals, and lines represent a linear regression of each treatment group. Inset plots are log-log transformed axes, with regression lines representing scaling of metabolic attributes with fish mass. $D-F$, Effect of developmental oxygen treatment (normoxia, hypoxia) and $\mathrm{Mo}_{2} \mathrm{DO}$ measurement level $(100 \% \mathrm{DO}, 50 \% \mathrm{DO})$ on $\mathrm{Mo}_{2 \min }(D), \mathrm{Mo}_{2 \max }(E)$, and aerobic scope $(F)$. There was no difference in $\mathrm{Mo}_{2 \min }, \mathrm{Mo}_{2 \text { max }}$, or aerobic scope between developmental treatment groups within the $100 \%$ and $50 \%$ DO measurement levels. Additionally, $\mathrm{Mo}_{2}$ min was not affected by $\dot{\mathrm{MO}}_{2} \mathrm{DO}$ measurement level. However, $\dot{\mathrm{M}}_{2 \text { max }}$ and aerobic scope were higher in the 100\% DO measurement group $(P<0.001)$. Thick horizontal lines are medians, boxes denote twenty-fifth and seventy-fifth percentiles, whiskers extend to the highest or lowest values within $1.5 \times$ the interquartile range, and filled circles are outliers beyond that range. Open circles indicate means.

to normal conditions (Burggren and Reyna 2011). While our results cannot address whether the metabolic phenotype was unchanged during development or was affected and subsequently returned to normal, evidence for plasticity of $\dot{\mathrm{MO}}_{2}$ in salmonids during and following developmental hypoxia exposure is mixed. The $\mathrm{Mo}_{2}$ min of rainbow trout embryos was not affected dur- ing developmental hypoxia exposure in one study (Miller et al. 2008), but recent work suggests an increase in $\dot{M}_{2}$ min in response to developmental hypoxia in Atlantic salmon alevins (Polymeropoulos 2013). A longer-term study did not measure $\dot{\mathrm{MO}}_{2}$ but found that maximum sustainable swimming speed $\left(U_{\text {crit }}\right)$ of rain- 


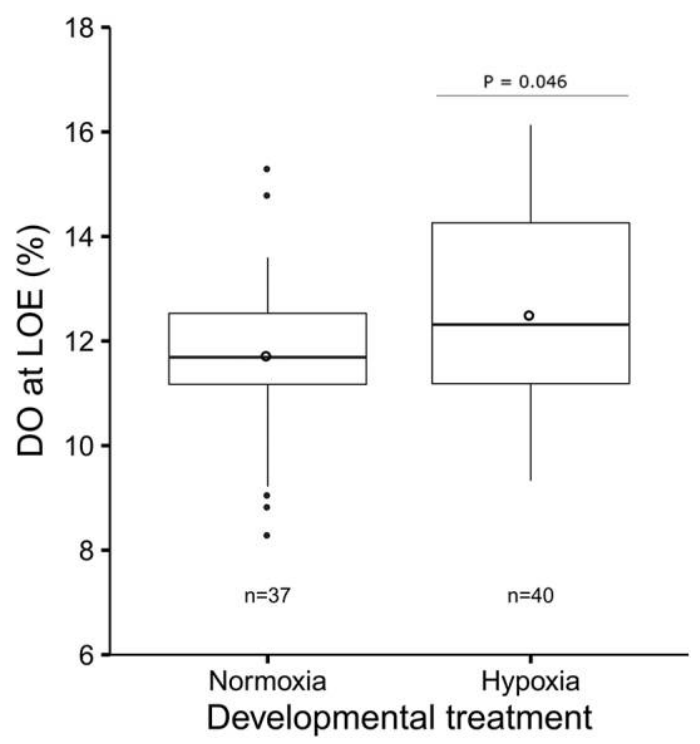

Figure 2. Effect of developmental oxygen treatment (normoxia, hypoxia) on dissolved oxygen (DO; \%) at loss of equilibrium (LOE). The DO at LOE of the hypoxia developmental treatment group was higher than for the treatment group incubated in normoxia $(P=$ 0.046). Thick horizontal lines are medians, boxes denote twenty-fifth and seventy-fifth percentiles, whiskers extend to the highest or lowest values within $1.5 \times$ the interquartile range, and filled circles are outliers beyond that range. Open circles indicate means.

bow trout was lower for up to $48 \mathrm{~d}$ following developmental hypoxia (Johnston et al. 2013). Also, developmental hypoxia has been shown to affect blood properties, heart rate programming, and muscle development in salmonids, although the longevity of these changes is unknown (Matschak et al. 1997; Miller et al. 2011; Bianchini and Wright 2013). Given the evidence for cardiovascular plasticity in salmonids exposed to developmental hypoxia, it is possible that fish exposed to developmental hypoxia in our study underwent plastic changes during development but transitioned back to a normal phenotype once returned to normoxic conditions. Moreover, it is unclear whether hypoxia during critical windows of embryonic and larval development or at other postlarval life stages may be more influential to long-term phenotypic trajectories than chronic hypoxia during embryonic and larval incubation (Burggren and Reyna 2011).

We measured a marked decrease in $\dot{M}_{2 \max }$ (50\%) and aerobic scope $(70 \%)$ but not $\dot{M}_{2 \text { min }}$ in both developmental groups when measured in acute hypoxia (50\% DO, $18 \mathrm{~h}$ ) compared with normoxic conditions (fig. 1). Since the $\mathrm{Mo}_{2 \text { min }}$ of fish in our study was not restricted at $50 \% \mathrm{DO}$, this provides evidence that the $\mathrm{O}_{2 \text { crit }}$ was below $50 \%$ DO. Indeed, $\mathrm{O}_{2 \text { crit }}$ has been reported as approximately $39 \% \mathrm{DO}$ at $12^{\circ} \mathrm{C}$ in Atlantic salmon (Remen et al. 2013) and $13.1 \% \mathrm{DO}$ at $10^{\circ} \mathrm{C}$ in rainbow trout (Ott et al. 1980). The $\mathrm{Mo}_{2 \max }$ decreased by $50 \%$ in $50 \%$ DO compared with normoxia (fig. $1 B, 1 E$ ), which is similar to a study of juvenile rainbow trout (Svendsen et al. 2012). Interestingly, it has been shown recently that $\dot{\mathrm{Mo}}_{2 \max }$ is affected differently in individuals exposed to hypoxia, depending on their $\dot{\mathrm{M}}_{2 \text { max }}$ in normoxia. Fish with a relatively low $\dot{\mathrm{MO}}_{2 \text { max }}$ are proportionally less affected than those with a high $\dot{M}_{2}$ max (Norin et al. 2016).

The relationships between DO at LOE and each of the metabolic attributes (fig. 3) are likely to be driven by how $\dot{M}_{2}$ max and $\dot{\mathrm{MO}}_{2 \text { min }}$ interact to determine $\mathrm{O}_{2 \text { crit }}$. Indeed, $\mathrm{O}_{2 \text { crit }}$ is thought to play a role in determining DO at LOE. The $\mathrm{O}_{2 \text { crit }}$ of an individual is often defined as the oxygen level below which $\dot{M}_{2}$ min
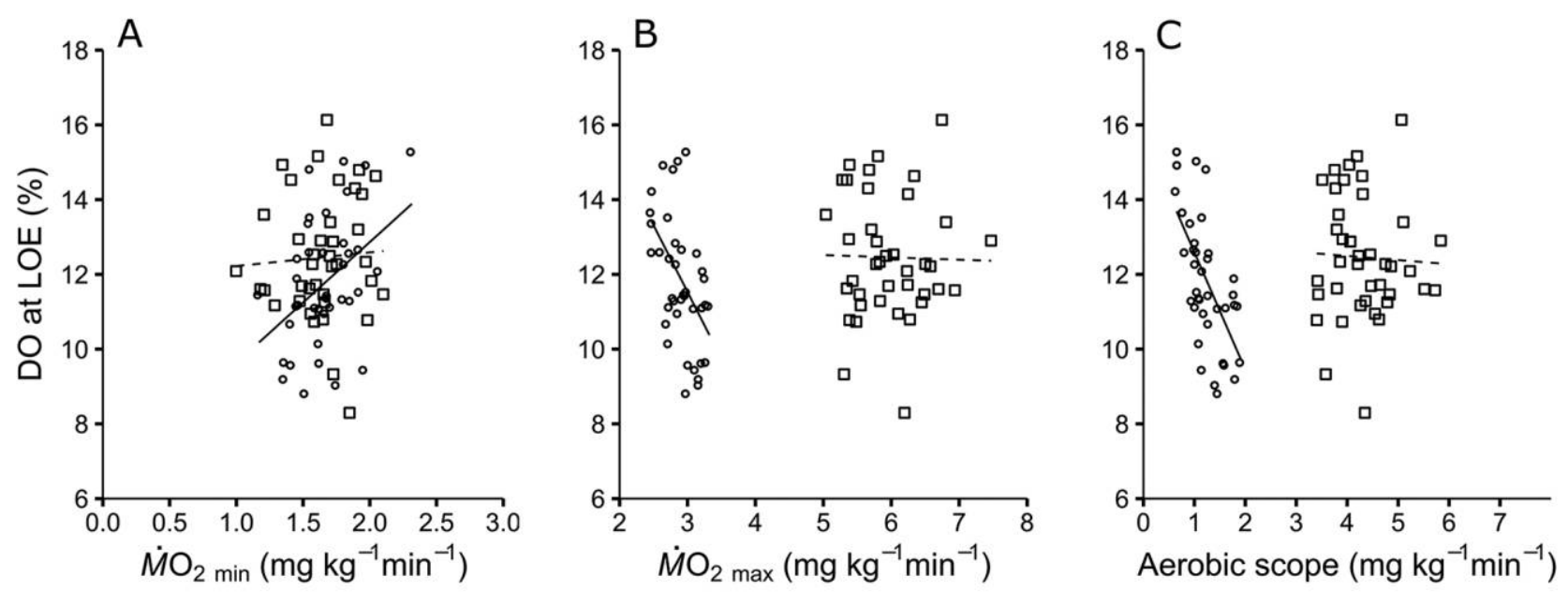

$$
\dot{M} \mathrm{O}_{2} \mathrm{DO} \div 50 \% \text { DO - }-100 \% \text { DO }
$$

Figure 3. Relationship between metabolic attributes $\dot{\mathrm{Mo}}_{2 \min }(A), \dot{\mathrm{Mo}}_{2 \max }(B)$, and aerobic scope $(C)$ with dissolved oxygen (DO; \%) at loss of equilibrium (LOE) for each $\dot{\mathrm{MO}}_{2}$ DO level (100\% DO, $50 \%$ DO). In $50 \%$ DO, relationships exist between DO at LOE and $\dot{\mathrm{MO}}_{2}$ min $(y=3.23 x+6.40)$, $\dot{\mathrm{MO}}_{2 \max }(y=-3.57 x+22.25)$, and aerobic scope $(y=-3.17 x+15.73)$. In $100 \% \mathrm{DO}$, there was no relationship between $\mathrm{DO}$ at $\mathrm{LOE}$ and $\dot{\mathrm{MO}}_{2 \mathrm{~min}}$, $\dot{\mathrm{MO}}_{2 \text { max }}$, and aerobic scope $(P>0.73$ in all cases). Data points represent individuals, and lines represent a linear regression of each treatment group. 
can no longer be maintained and fish begin oxyconforming. Similarly, $\mathrm{O}_{2}$ crit is also thought to coincide with the oxygen level where declining $\dot{\mathrm{M}}_{2 \text { max }}$ intersects with $\dot{\mathrm{M}}_{2 \text { min }}$, which implicates both metabolic parameters in determining $\mathrm{O}_{2 \text { crit }}$ (Claireaux et al. 2000; Svendsen et al. 2012; Claireaux and Chabot 2016). Individual salmon in our study exhibited a lower DO at LOE when they possessed a higher $\dot{\mathrm{M}}_{2 \text { max }}$ and aerobic scope and a lower $\dot{\mathrm{Mo}}_{2 \text { min }}$, suggesting that $\mathrm{O}_{2 \text { crit }}$ is dependent on $\dot{\mathrm{Mo}}_{2 \text { max }}$ and $\dot{\mathrm{MO}}_{2 \text { min }}$ and contributes to driving the threshold level of DO before LOE. However, this pattern was evident only for $\dot{M}_{2}$ max measured in hypoxic conditions. This may be because $\dot{M}_{2}$ max is not necessarily limited by oxygen availability in normoxic conditions, and therefore interindividual differences in the capacity of fish to take up oxygen may not be fully revealed when measuring $\dot{M}_{2}$ max in normoxia (Lefrançois and Claireaux 2003; Svendsen et al. 2012). However, limited oxygen availability does not explain why we found a relationship between $\mathrm{Mo}_{2 \text { min }}$ at $50 \%$ DO and DO at LOE but not for $\dot{\mathrm{MO}}_{2 \text { min }}$ at $100 \%$ DO, because $\dot{\mathrm{MO}}_{2 \text { min }}$ is not reduced until below $\mathrm{O}_{2 \text { crit. }}$. An explanation for this may be that individual fish have differential responses to environmental fluctuations, depending on the trait being measured, as seen in barramundi (Lates calcarifer; Norin et al. 2016). Nevertheless, because we did not measure the response of each individual in both normoxia and hypoxia, it remains uncertain whether $\dot{M}_{2}$ min measurements in hypoxia truly reveal a differential relationship between $\dot{M}_{2}$ min and DO at LOE at the individual level.

We found that developmental hypoxia had a negligible effect on the long-term aerobic performance and hypoxia tolerance of Atlantic salmon, despite evidence of immediate and enduring cardiovascular alterations in response to developmental hypoxia in other fish species (Yaqoob and Schwerte 2010; Miller et al. 2011; Johnston et al. 2013). As hypoxic conditions continue to become more prevalent in aquatic systems (Diaz and Rosenberg 2008; Altieri and Gedan 2015), it is increasingly important to understand the likely intra- and interspecific responses of aquatic taxa. Future research will help to understand whether developmental hypoxia has immediate and universal effects on organismal performance and, if so, how long these changes are sustained once benign conditions are reestablished.

\section{Acknowledgments}

We would like to thank the SALTAS hatchery staff for supplying eggs and assisting in fish rearing. All experiments carried out in this study were performed in compliance with the University of Tasmania Animal Ethics Committee (Hobart, Australia) under ethics permit A0013794.

\section{Literature Cited}

Altieri A.H. and K.B. Gedan. 2015. Climate change and dead zones. Glob Chang Biol 21:1395-1406.
Anttila K., R.S. Dhillon, E.G. Boulding, A.P. Farrell, B.D. Glebe, J.A.K. Elliott, W.R. Wolters, and P.M. Schulte. 2013. Variation in temperature tolerance among families of Atlantic salmon (Salmo salar) is associated with hypoxia tolerance, ventricle size and myoglobin level. LExp Biol 216:1183-1190.

Bagatto B. 2005. Ontogeny of cardiovascular control in zebrafish (Danio rerio): effects of developmental environment. Comp Biochem Physiol A 141:391-400.

Bianchini K. and P.A. Wright. 2013. Hypoxia delays hematopoiesis: retention of embryonic hemoglobin and erythrocytes in larval rainbow trout, Oncorhynchus mykiss, during chronic hypoxia exposure. LExp Biol 216:4415-4425.

Burggren W.W. and K.S. Reyna. 2011. Developmental trajectories, critical windows and phenotypic alteration during cardiorespiratory development. Respir Physiol Neurobiol 178:13-21.

Burt K., D. Hamoutene, G. Mabrouk, C. Lang, T. Puestow, D. Drover, R. Losier, and F. Page. 2012. Environmental conditions and occurrence of hypoxia within production cages of Atlantic salmon on the south coast of Newfoundland. Aquac Res 43:607-620.

Chabot D., J.F. Steffensen, and A.P. Farrell. 2016. The determination of standard metabolic rate in fishes. L Fish Biol $88: 81-121$.

Ciuhandu C.S., P.A. Wright, J.I. Goldberg, and E.D. Stevens. 2007. Parameters influencing the dissolved oxygen in the boundary layer of rainbow trout (Oncorhynchus mykiss) embryos and larvae. LExp Biol 210:1435-1445.

Claireaux G. and D. Chabot. 2016. Responses by fishes to environmental hypoxia: integration through Fry's concept of aerobic metabolic scope. I Fish Biol 88:232-251.

Claireaux G., D.M. Webber, J.P. Lagardère, and S.R. Kerr. 2000. Influence of water temperature and oxygenation on the aerobic metabolic scope of Atlantic cod (Gadus morhua). I Sea Res 44:257-265.

Clark T.D., E. Sandblom, and F. Jutfelt. 2013. Aerobic scope measurements of fishes in an era of climate change: respirometry, relevance and recommendations. LExp Biol 216:2771-2782.

Dejours P. 1981. Principles of comparative respiratory physiology. Elsevier, Amsterdam.

Dhiyebi H.A., M.J. O’Donnell, and P.A. Wright. 2013. Water chemistry in the microenvironment of rainbow trout Oncorhynchus mykiss embryos is affected by development, the egg capsule and crowding. L Fish Biol 82:444-457.

Diaz R.J. and R. Rosenberg. 2008. Spreading dead zones and consequences for marine ecosystems. Science 321:926-929.

Garland T., Jr., M.D. Cadney, and R.A. Waterland. 2017. Early-life effects on adult physical activity: concepts, relevance, and experimental approaches. Physiol Biochem Zool 90:1-14.

Greig S.M., D.A. Sear, and P.A. Carling. 2007. A review of factors influencing the availability of dissolved oxygen to incubating salmonid embryos. Hydrol Process 21:323-334.

Ingendahl D. 2001. Dissolved oxygen concentration and emergence of sea trout fry from natural redds in tributaries of the River Rhine. LFish Biol 58:325-341.

Johansson D., K. Ruohonen, A. Kiessling, F. Oppedal, J.-E. Stiansen, M. Kelly, and J.-E. Juell. 2006. Effect of environ- 
mental factors on swimming depth preferences of Atlantic salmon (Salmo salar L.) and temporal and spatial variations in oxygen levels in sea cages at a fjord site. Aquaculture 254: 594-605.

Johnston E.F., S.L. Alderman, and T.E. Gillis. 2013. Chronic hypoxia exposure of trout embryos alters swimming performance and cardiac gene expression in larvae. Physiol Biochem Zool 86:567-575.

Lefrançois C. and G. Claireaux. 2003. Influence of ambient oxygenation and temperature on metabolic scope and scope for heart rate in the common sole Solea solea. Mar Ecol Prog Ser 259:273-284.

Matschak T.W., N.C. Stickland, P.S. Mason, and A.R. Crook. 1997. Oxygen availability and temperature affect embryonic muscle development in Atlantic salmon (Salmo salar L.). Differentiation 61:229-235.

McLean W.E. and P.G. Lim. 1985. Some effects of water flow rate and alevin density in vertical incubation trays on early development of chinook salmon (Oncorhynchus tshawytscha). Canadian Technical Report of Fisheries and Aquatic Sciences no. 1357. Department of Fisheries and Oceans, Vancouver.

Metcalfe N.B., T.E. Van Leeuwen, and S.S. Killen. 2016. Does individual variation in metabolic phenotype predict fish behaviour and performance? I Fish Biol 88:298-321.

Miller S.C., T.E. Gillis, and P.A. Wright. 2011. The ontogeny of regulatory control of the rainbow trout (Oncorhynchus mykiss) heart and how this is influenced by chronic hypoxia exposure. LExp Biol 214:2065-2072.

Miller S.C., S.E. Reeb, P.A. Wright, and T.E. Gillis. 2008. Oxygen concentration in the water boundary layer next to rainbow trout (Oncorhynchus mykiss) embryos is influenced by hypoxia exposure time, metabolic rate, and water flow. Can J Fish Aquat Sci 65:2170-2177.

Monaghan P. 2008. Early growth conditions, phenotypic development and environmental change. Philos Trans R Soc B 363: 1635-1645.

Mueller C.A., J. Eme, W.W. Burggren, R.D. Roghair, and S.D. Rundle. 2015. Challenges and opportunities in developmental integrative physiology. Comp Biochem Physiol A 184:113-124.

Norin T. and T.D. Clark. 2016. Measurement and relevance of maximum metabolic rate in fishes. IFish Biol 88:122-151.

Norin T., H. Malte, and T.D. Clark. 2014. Aerobic scope does not predict the performance of a tropical eurythermal fish at elevated temperatures. LExp Biol 217:244-251.

- 2016. Differential plasticity of metabolic rate phenotypes in a tropical fish facing environmental change. Funct Ecol 30:369-378.

Oppedal F., T. Dempster, and L.H. Stien. 2011. Environmental drivers of Atlantic salmon behaviour in sea-cages: a review. Aquaculture 311:1-18.
Ott M.E., N. Heisler, and G.R. Ultsch. 1980. A re-evaluation of the relationship between temperature and the critical oxygen tension in freshwater fishes. Comp Biochem Physiol A 67:337-340.

Pedersen C.L. 1987. Energy budgets for juvenile rainbow trout at various oxygen concentrations. Aquaculture 62:289-298.

Pelster B. 2002. Developmental plasticity in the cardiovascular system of fish, with special reference to the zebrafish. Comp Biochem Physiol A 133:547-553.

Polymeropoulos E.T. 2013. The effects of oxygen and temperature on the physiology of hatching stage Atlantic salmon. PhD diss. University of Tasmania, Hobart.

R Development Core Team. 2015. R: a language and environment for statistical computing. R Foundation for Statistical Computing, Vienna. https://www.R-project.org/.

Reidy S.P., S.R. Kerr, and J.A. Nelson. 2000. Aerobic and anaerobic swimming performance of individual Atlantic cod. LExp Biol 203:347-357.

Remen M., F. Oppedal, A.K. Imsland, R.E. Olsen, and T. Torgersen. 2013. Hypoxia tolerance thresholds for postsmolt Atlantic salmon: dependency of temperature and hypoxia acclimation. Aquaculture 416/417:41-47.

Robertson C.E., P.A. Wright, L. Köblitz, and N.J. Bernier. 2014. Hypoxia- inducible factor-1 mediates adaptive developmental plasticity of hypoxia tolerance in zebrafish, Danio rerio. Proc R Soc B 281:20140637.

Rombough P.J. 2007. Oxygen as a constraining factor in egg size evolution in salmonids. Can I Fish Aquat Sci 64:692699.

Svendsen J.C., J.F. Steffensen, K. Aarestrup, M. Frisk, A. Etzerodt, and M. Jyde. 2012. Excess posthypoxic oxygen consumption in rainbow trout (Oncorhynchus mykiss): recovery in normoxia and hypoxia. Can I Zool 90:1-11.

Vanderplancke G., G. Claireaux, P. Quazuguel, L. Madec, S. Ferraresso, A. Sévère, J.-L. Zambonino-Infante, and D. Mazurais. 2014. Hypoxic episode during the larval period has long-term effects on European sea bass juveniles (Dicentrarchus labrax). Mar Biol 162:367-376.

Wang T., S. Lefevre, D.T.T. Huong, N.V. Cong, and M. Bayley. 2009. The effects of hypoxia on growth and digestion. Pp. 361-396 in J.G. Richards, A.P. Farrell, and C.J. Brauner, eds. Fish physiology. Academic Press, New York.

Yaqoob N. and T. Schwerte. 2010. Cardiovascular and respiratory developmental plasticity under oxygen depleted environment and in genetically hypoxic zebrafish (Danio rerio). Comp Biochem Physiol A 156:475-484.

Youngson A.F., I.A. Malcolm, J.L. Thorley, P.J. Bacon, and C. Soulsby. 2004. Long-residence groundwater effects on incubating salmonid eggs: low hyporheic oxygen impairs embryo development. Can I Fish Aquat Sci 61:2278-2287. 